\title{
VIABILIDADE ECONÔMICA DA TERMINAÇÃO DE NOVILHOS NELORE E RED NORTE EM CONFINAMENTO NÁ REGIÃO DE LAVRAS-MG
}

\author{
Economical viability of finishing Nellore and Red Norte bulls in feedlot, in Lavras-MG region
}

\author{
Leandro Sâmia Lopes ${ }^{1}$, Márcio Machado Ladeira ${ }^{1}$, Otávio Rodrigues Machado Neto ${ }^{2}$, \\ Antonio Rogério Mendes Carli da Silveira ${ }^{1}$, Ricardo Pereira Reis ${ }^{3}$, Fabrício Rodrigues Campos $^{4}$
}

\begin{abstract}
RESUMO
Conduziu-se este trabalho, com o objetivo de verificar a viabilidade econômica da terminação em confinamento de novilhos Nelore e Red Norte na região de Lavras, MG. Foram utilizados 41 novilhos, não castrados, de dois grupos genéticos, sendo: 19 Nelore e 22 Red Norte, com pesos vivos iniciais médios de $315 \pm 31 \mathrm{~kg}$ e $321 \pm 30 \mathrm{~kg}$, respectivamente. O período experimental foi de 112 dias, divididos em quatro períodos de 28 dias. A viabilidade econômica foi analisada utilizando o modelo de custo, considerando os custos operacionais, alternativos e de depreciação. Todos os indicadores econômicos gerados apresentaram resultados negativos. A atividade apresentou prejuízo de $\mathrm{R} \$ 6,88$ e 19,74/arroba para os animais Red Norte e Nelore, respectivamente. Os resultados negativos obtidos nessas condições ocorreram porque o custo operacional efetivo foi maior que o preço de venda da arroba. Em um cenário com a aquisição de animais a atividade de confinamento apresentou resíduo próximo de zero para o grupo Red Norte. A prática do confinamento não apresentou viabilidade econômica para a região de Lavras, MG, no ano de 2007.
\end{abstract}

Termos para indexação: Economia rural, gestão, pecuária de corte, sistemas de produção.

\begin{abstract}
The objective was to study the economic viability of feedlot in Lavras region, state of Minas Gerais. Forty one bulls of two genetic groups were used, being: 19 Nellore and 22 Red Norte, with initial average age of 20 months and initial average live weight of $315 \pm 31 \mathrm{~kg}$ and $321 \pm 30 \mathrm{~kg}$, respectively. The experimental period was 112 days. The model of cost was used to evaluate the economic viability. All economic indexes presented negative results. The activity presented negative profit of $\mathrm{R} \$ 6.88$ and $\mathrm{R} \$ 1$ 19.74/arroba for the animals Red Norte and Nellore, respectively. The negative results obtained in these conditions took place because the operational effective cost was bigger than the sale price of the arroba. The feedlot didn't present economical viability for the Lavras region, in the year of 2007. The feed efficiency has straight influence on the economical viability of feedlot.
\end{abstract}

Index terms: Beef cattle production, management, production systems, rural economy.

(Recebido em 25 de março de 2010 e aprovado em 4 de fevereiro de 2011)

\section{INTRODUÇÃo}

O Brasil possui o segundo maior rebanho bovino mundial, com aproximadamente 207 milhões de cabeças (INSTITUTO BRASILEIRO DE GEOGRAFIA E ESTATÍSTICA - IBGE, 2006), sendo o primeiro rebanho comercial do mundo. No ano de 2003, o país se consolidou como o maior exportador mundial de carne bovina, ultrapassando a Austrália. Todavia, a produtividade da pecuária de corte nacional ainda se encontra aquém à dos principais países concorrentes. Segundo Fávare (2008), a taxa de desfrute nacional se encontra em $22 \%$, enquanto que nos Estados Unidos essa taxa chega a 37\%. Portanto, estratégias de intensificação da produção de carne no país, com a utilização de genótipos melhorados e a redução da idade de abate, podem contribuir para elevar a taxa de desfrute da pecuária de corte brasileira.

Também se deve considerar que nos últimos anos o mercado da carne no Brasil e no mundo vem se tornando cada vez mais competitivo e complexo. Isso ocorre, em razão da concorrência entre as diferentes carnes (bovina, suína e de aves), do ciclo de vida e do grau de perecibilidade do produto, das exigências por rigorosos controles sanitários e da crescente demanda por diversificação e agregação de valor (CAMARGO et al., 2008). Ademais, existe necessidade de geração de renda para a maior sustentabilidade econômica da atividade (AMARAL, 2010). Diante desses fatores, fica demonstrada a importância

\footnotetext{
1Universidade Federal de Lavras/UFLA - Departamento de Zootecnia/DZO - Lavras, MG

²Universidade Federal de Lavras/UFLA - Departamento de Zootecnia/DZO - Cx. P. 3037 - 3200-000 - Lavras, MG - otavionetto@terra.com.br

3Universidade Federal de Lavras/UFLA - Departamento de Administração e Economia/DAE - Lavras, MG

${ }^{4}$ Mestre em Zootecnia
} 
cada vez maior da produção eficiente, que além de ser caracterizada pela redução do ciclo de produção, exige produtos de melhor qualidade e que tragam um maior retorno econômico (BONILHA, 2007).

Quando se leva em consideração a necessidade de redução da idade de abate dos animais e/ou ciclos de produção mais curtos, pode-se utilizar como alternativa o confinamento. Entretanto, segundo Barbosa e Souza (2007), com a intensificação dos sistemas de produção ocorre aumento dos custos operacionais variáveis, necessitando de maiores desembolsos no fluxo de caixa da empresa rural. Por outro lado, o aumento da produção tende a diluir os custos operacionais fixos.

A nutrição é um dos fatores de maior importância em sistemas de produção de bovinos, principalmente em situações onde os animais se encontram confinados, pois nesse sistema, o custo com alimentação tem grande impacto sobre o custo total da atividade. $\mathrm{O}$ conhecimento dos custos permite a análise econômica da atividade e por meio dessa conhecer com detalhes os fatores de produção, como: terra, capital e trabalho (LOPES; MAGALHÃES, 2005). Atualmente, tem sido crescente o número de artigos publicados que avaliaram os custos de produção na pecuária de corte (FERREIRA et al., 2004; OLIVEIRA et al., 2007), sendo que esse tipo de avaliação em sistemas de confinamento de bovinos passou a ter grande importância nos últimos anos em razão do grande crescimento dessa atividade no país.

Neste trabalho, objetivou-se verificar a viabilidade econômica da terminação em confinamento de novilhos Nelore e Red Norte, na região de Lavras, sul do estado de Minas Gerais.

\section{MATERIAL E MÉTODOS}

O estudo foi desenvolvido no Departamento de Zootecnia da Universidade Federal de Lavras, em Minas
Gerais, no período de junho a outubro de 2007. Foram utilizados 41 novilhos, não castrados, de dois grupos genéticos, sendo: 19 Nelore, com peso vivo inicial médio de $315 \pm 31 \mathrm{~kg}$ e 22 Red Norte ( $1 / 2$ Senepol, $1 / 4$ Caracu, $1 / 4$ F1 Angus-Nelore) com peso vivo inicial médio de $321 \pm 30 \mathrm{~kg}$. A idade inicial média de ambos os grupos genéticos era de 20 meses. Os animais foram confinados em baias coletivas com área de $30 \mathrm{~m}^{2}$ por animal, separados de acordo com o grupo genético.

O período experimental teve duração de 112 dias, sendo que este foi composto por quatro períodos de 28 dias. No início do experimento e ao final de cada período foi realizada a pesagem dos animais após jejum de 16 horas.

A dieta (Tabela 1) foi balanceada para atender às exigências de acordo com o National Research Council NRC (2000) (14,3\% de PB, 70,3\% de NDT e $30,1 \%$ de FDNcp) e foi fornecida ad libitum aos animais às $08 \mathrm{~h} 00 \mathrm{e}$ $15 \mathrm{~h} 00$ em forma de ração completa. Na Tabela 1, também são apresentados os preços dos ingredientes na matéria seca e o custo total da dieta fornecida.

O consumo de matéria seca (CMS) foi obtido individualmente por meio do uso de indicadores. Para a estimação da produção fecal, utilizou-se o indicador LIPE $^{\circledR}$, numa posologia de uma cápsula de $0,5 \mathrm{~g} / \mathrm{animal} / \mathrm{dia}$ (RODRIGUEZ et al., 2007), durante os dias 24, 25 e 26 de cada período experimental.

Para determinação do consumo de concentrado foi utilizado o óxido crômico misturado ao concentrado na proporção de $10 \mathrm{~g} / \mathrm{animal} / \mathrm{dia}$ e fornecido aos animais entre o 19 o e 27으 dia de dois períodos experimentais (segundo e terceiro). Para o consumo do volumoso, utilizou-se a MSi, obtida após 240 horas de incubação ruminal. Durante todos os dias de fornecimento do óxido crômico, foram coletadas amostras do volumoso e concentrado.

Tabela 1 - Composição percentual da dieta, preços dos ingredientes na matéria seca e custo total da dieta fornecida.

\begin{tabular}{lccc}
\hline Ingredientes $^{1}$ & $(\%)$ & Preço dos Ingredientes (R\$) em kg de MS & Sub-total \\
\hline Silagem de milho & 50,0 & 0,15 & 0,08 \\
Milho & 23,0 & 0,44 & 0,10 \\
Polpa Cítrica & 11,5 & 0,49 & 0,06 \\
Farelo de Soja 46\% PB & 10,0 & 0,69 & 0,07 \\
Farelo de Algodão 28\% PB & 3,4 & 0,60 & 0,02 \\
Núcleo Mineral & 1,6 & 0,20 & 0,00 \\
Uréia & 0,5 & 1,67 & 0,01 \\
\hline Preço total de um kg de MS de dieta $(\mathrm{R} \$)$ & & & 0,33 \\
\hline 1\% da Matéria Seca. & &
\end{tabular}


Como as estimativas do CMS individual foram realizadas apenas no segundo e terceiro períodos experimentais, fez-se a extrapolação desses dados para todo o experimento (112 dias) com base no CMS expresso em porcentagem do peso vivo.

Para o estudo da análise econômica, o primeiro passo foi realizar o inventário de todos os materiais, equipamentos e instalações necessários para o desenvolvimento da atividade de um confinamento para 120 animais e seus respectivos preços. Nessa situação, a implantação de um confinamento teria um custo inicial de aproximadamente $\mathrm{R} \$ 70.000,00$. O custo de um trator e do vagão forrageiro representaram os principais componentes do investimento. Posteriormente, foram calculados os diferentes indicadores econômicos (REIS et al., 2002). Considerou-se o valor de depreciação fracionado para um período de 20 anos e foi estabelecido um custo alternativo do capital investido equivalente aos juros pagos pela poupança (6\% a.a.).

Para determinação dos custos por ciclo de produção (112 dias de experimento) foi necessário calcular a depreciação e o custo alternativo diário e então multiplicar pelo período de 112 dias. Esses cálculos foram feitos segundo as equações abaixo:

\section{$\mathrm{D}=(\underline{\mathrm{Va}-\mathrm{Vr}) / \mathrm{Vu}} \times 112$, 365}

onde: $\mathrm{D}=$ depreciação por ciclo de confinamento de 112 dias;

$\mathrm{Va}=$ valor de aquisição do bem;

$\mathrm{Vr}=$ valor residual (taxa de $10 \%$ do $\mathrm{Va}$ );

$\mathrm{Vu}=$ vida útil

$$
\mathrm{CA}=\frac{\mathrm{Va} \times \mathrm{Taxa} \text { de juros }(6 \% \mathrm{a} . \mathrm{a})}{365} \times 112
$$

onde: $\mathrm{CA}=$ custo alternativo por ciclo de confinamento de 112 dias

$\mathrm{Va}=$ valor de aquisição do bem

Os cálculos de depreciação e de custo alternativo obtidos levaram em consideração a capacidade total instalada do confinamento que é de 120 animais. Portanto, ambos os valores foram divididos por 120 , obtendo-se assim, os custos por animal.

O cálculo do custo operacional efetivo considerou os gastos com mão-de-obra, combustível, sanidade e alimentação, que foi determinado por meio do consumo real da dieta e uma sobra estimada de $2 \%$. Esse procedimento, para determinação do custo de alimentação, simula as condições de confinamentos empresariais.

Para o cálculo dos custos com mão-de-obra foi considerado o trabalho de um operador de trator e de um ajudante, ambos recebendo uma diária no valor de vinte reais ( $\mathrm{R} \$ 20,00)$, e que estes são capazes de fornecer a dieta para 500 animais. Posteriormente, esse gasto foi individualizado por animal, assim como foi realizado para a depreciação e o custo alternativo.

$\mathrm{O}$ custo operacional total foi obtido somando o valor descrito no custo operacional efetivo mais o valor decorrente da depreciação dos materiais, instalações e equipamentos necessários para a condução do confinamento.

O custo total foi resultado da soma do custo operacional total, com o custo alternativo do capital investido em materiais, instalações e equipamentos.

A renda bruta (RB) foi obtida levando em consideração o preço de venda de arroba vigente na época para a região de Lavras-MG, que foi de $\mathrm{R} \$ 60,00$.

$\mathbf{R B}=\mathbf{n}^{\mathbf{0}}$ arrobas obtidas nos 112 dias $x \mathbf{R} \$ \mathbf{6 0 , 0 0}$.

A partir da metodologia descrita acima foram gerados os seguintes indicadores econômicos:

Margem Bruta $=$ Receita Total - Custo Operacional Efetivo;

Margem Líquida $=$ Receita Total - Custo Operacional

Total;

Resíduo = Receita Total - Custo Total.

Lucratividade $=$ Margem Líquida $/$ Receita Total $x 100$

\section{RESULTADOS E DISCUSSÃO}

$\mathrm{Na}$ Tabela 2, são apresentados a renda bruta, os custos de produção e os indicadores financeiros da terminação dos 41 animais em um sistema em que os animais pertencem à empresa, não havendo necessidade de aquisição.

Os animais Red Norte e Nelore apresentaram ganho de peso médio diário de 1,81 e 1,43 kg/dia, respectivamente. Portanto, ao final do período experimental o grupo Red Norte obteve quase uma arroba a mais de peso vivo. Esse desempenho refletiu em uma renda bruta de $\mathrm{R} \$ 394,80$ e $\mathrm{R} \$$ 338,40/animal, respectivamente.

As despesas operacionais referentes aos gastos com alimentação, mão-de-obra, sanidade e combustível totalizaram por arroba a quantia de $\mathrm{R} \$ 64,76$ para o grupo Red Norte e R \$77,27 para a raça Nelore. Pode-se verificar que a alimentação representou cerca de $87 \%$ do custo total da atividade de confinamento. Segundo Restle e Vaz (1999), no processo de terminação em confinamento a alimentação geralmente representa mais de $70 \%$ do custo total de 
produção. Pacheco et al. (2006), ao avaliarem a eficiência econômica dos grupos genéticos 5/8 Charolês-3/8 Nelore e 5/8 Nelore-3/8 Charolês, verificaram que a alimentação representou cerca de $74 \%$ do custo total do confinamento. Nogueira (2006) apresentou estimativas de custos totais para o confinamento no estado de São Paulo no ano de 2006, em que a alimentação representou de 75,8 a 80,9\% do custo total desta atividade. Segundo Lopes et al. (2005), em um confinamento de 1.000 animais, 95,6\% dos custos diretos (excluindo a compra de animais) seria relativo à alimentação. Portanto, os resultados encontrados neste trabalho se aproximam do relato deste último autor e se distanciam dos valores descritos por Restle e Vaz (1999) e Pacheco et al. (2006).

Os custos calculados com a depreciação de equipamentos, materiais e instalações foram de $\mathrm{R} \$ 8,02$ por animal. Já, o custo alternativo representou $\mathrm{R} \$ 10,69$ por animal para um período de 112 dias de confinamento (Tabela 2). Observa-se, portanto, que os custos com depreciação e custo alternativo (1,80 e 2,41\%, respectivamente, para o grupo Red Norte) tiveram pouco impacto sobre o custo final da arroba, o que demonstra que em um sistema como este, o empresário deve se preocupar principalmente com os custos de alimentação e

Tabela 2 - Renda Bruta, Custos de Produção e Indicadores Financeiros do confinamento de novilhos Red Norte e Nelore.

\begin{tabular}{|c|c|c|c|c|c|c|}
\hline \multirow{3}{*}{ Especificação } & \multicolumn{3}{|c|}{ Red Norte } & \multicolumn{3}{|c|}{ Nelore } \\
\hline & \multicolumn{2}{|c|}{ Total } & \multirow{2}{*}{$\begin{array}{l}\% \text { do } \\
\text { Custo } \\
\text { Total }\end{array}$} & \multicolumn{2}{|c|}{ Total } & \multirow{2}{*}{$\begin{array}{c}\% \text { do } \\
\text { Custo } \\
\text { Total }\end{array}$} \\
\hline & $\begin{array}{c}\mathrm{R} \$ / \\
\text { Animal }\end{array}$ & $\begin{array}{c}\mathrm{R} \$ / \\
@ \text { produzida }\end{array}$ & & $\begin{array}{c}\mathrm{R} \$ / \\
\text { animal }\end{array}$ & $\begin{array}{c}\mathrm{R} \$ / \\
\text { @ produzida }\end{array}$ & \\
\hline \multicolumn{7}{|l|}{ 1. Renda Bruta (RB) } \\
\hline Renda bruta & 394,80 & 60 & & 338,40 & 60 & \\
\hline \multicolumn{7}{|l|}{ 2. Custo de Produção } \\
\hline \multicolumn{7}{|l|}{$\begin{array}{l}2.1 \text { Custo Operacional Efetivo } \\
\text { (CopE) }\end{array}$} \\
\hline Mão-de-obra & 8,96 & 1,36 & 2,01 & 8,96 & 1,59 & 1,97 \\
\hline Alimentação & 387,15 & 58,84 & 87,03 & 396,76 & 70,35 & 87,30 \\
\hline Combustível e Lubrificantes & 25,77 & 3,91 & 5,79 & 25,77 & 4,57 & 5,67 \\
\hline Sanidade & 4,27 & 0,65 & 0,96 & 4,27 & 0,76 & 0,94 \\
\hline Total do CopE & 426,15 & 64,76 & 95,79 & 435,76 & 77,27 & 95,88 \\
\hline \multicolumn{7}{|l|}{ 2.2. Custo Operacional Total } \\
\hline Custo Operacional Efetivo & 426,15 & 64,76 & 95,79 & 435,76 & 77,27 & 95,88 \\
\hline Depreciação & 8,02 & 1,22 & 1,80 & 8,02 & 1,42 & 1,76 \\
\hline Total do CopT & 434,17 & 65,98 & 97,59 & 443,78 & 78,69 & 97,64 \\
\hline \multicolumn{7}{|l|}{ 2.3. Custo Total (CT) } \\
\hline Custo Operacional Total & 434,17 & 65,98 & 97,59 & 443,78 & 78,69 & 97,64 \\
\hline Custo alternativo (C.A.) & 10,69 & 1,62 & 2,41 & 10,69 & 1,90 & 2,36 \\
\hline Custo Total (CT) & 444,86 & 67,60 & 100,00 & 454,47 & 80,59 & 100,00 \\
\hline \multicolumn{7}{|l|}{ 3. Indicadores Financeiros } \\
\hline 3.1 Margem Bruta (R.B.-CopE) & $-31,35$ & $-4,76$ & & $-97,36$ & $-17,27$ & \\
\hline 3.2 Margem Líquida (R.B.-CopT) & $-39,37$ & $-5,98$ & & $-105,38$ & $-18,69$ & \\
\hline $\begin{array}{l}\text { 3.3 Lucratividade (Margem } \\
\text { Líq/Receita Total) }\end{array}$ & $-9,97 \%$ & $-9,97 \%$ & & $-31,14 \%$ & $-31,14 \%$ & \\
\hline 3.4 Resíduo (R.B.-C.T) & $-50,06$ & $-7,60$ & & $-116,07$ & $-20,59$ & \\
\hline
\end{tabular}


com a busca por animais de alta eficiência alimentar. A baixa representatividade dos custos de depreciação e alternativo no custo total decorre do baixo investimento necessário para as instalações (piso de terra compactado, divisórias em arame liso, cocho do tipo vinilona e sem cobertura).

O menor custo operacional do confinamento de animais Red Norte, em relação a zebuínos demonstra a importância da correta escolha do grupo genético a ser utilizado na terminação. De acordo com Calegare et al. (2009), a raça mais eficiente em um ambiente de restrição nutricional pode não ter o melhor desempenho, quando em situações de farta disponibilidade de alimento (situação comum em confinamentos). Entretanto, é importante afirmar que quando a nutrição limita o desempenho, raças com maior exigência de energia podem apresentar baixo desempenho. Portanto, na determinação do grupo genético a ser utilizado, as condições em que os animais serão submetidos têm grande importância na lucratividade da atividade.

Todos os indicadores econômicos gerados apresentaram resultados negativos. A atividade apresentou prejuízo de R \$ 7,60 e R\$ 20,59/arroba para os animais Red Norte e Nelore, respectivamente. Os resultados negativos obtidos nestas condições podem ser explicados porque o custo operacional efetivo foi maior que o preço de venda (R\$60,00/@). Isso demonstra a importância de se conhecer o mercado e a necessidade de fixação de preços, quando se utiliza um sistema de produção de alto custo. As principais opções nesse caso são: contratos futuros na $\mathrm{BM} \& \mathrm{~F}$ e contratos de boi a termo com a indústria frigorífica. Além disso, no ano de 2007, houve grande elevação dos preços de alguns ingredientes para a alimentação animal, como o milho e a polpa cítrica. Como exemplo, o preço médio da saca de milho no ano de 2010 foi aproximadamente $35 \%$ menor do que os preços pagos por esse alimento na situação analisada. Isso demonstra, que para o sucesso da atividade de confinamento, dois pontos são fundamentais: preço de venda dos animais e custo de alimentação.

Oliveira et al. (2007) encontram que o confinamento, dependendo da dieta utilizada, apresentou Margem Bruta/ animal de R \$ -41,38 a R \$23,45. Sendo assim, a formulação de dietas de baixo custo, mas com valores nutricionais adequados, podem representar o retorno positivo.

Ao avaliarem o confinamento de diferentes grupos genéticos, Ferreira et al. (2004) encontram que no ano de 2002 a atividade apresentou os custos superiores às receitas. Todavia, no mesmo trabalho os autores fizeram uma análise com base no preço médio entre os anos de 1997 e 2002 e verificaram Margem Bruta positiva. Outro ponto importante deste trabalho foi a grande variação nas Margens Brutas dependendo do grupo genético submetido ao confinamento. Nesta última situação a variação foi de US\$ 6.38 a US\$ 94.56. Esses resultados se assemelham aos do presente estudo.

Em um cenário com a aquisição de animais (Tabela 3), percebe-se a grande influência desse gasto sobre o custo total de produção. Ao analisar os resultados, observa-se que se o confinamento fosse conduzido apenas com animais Red Norte, o resíduo seria próximo de zero, o que indica ausência de lucro e/ou prejuízo. Isso ocorreu, em razão do ganho de receita decorrente da valorização da arroba no período. Ao adquirir os animais, a arroba se encontrava no preço de $\mathrm{R} \$ 55,00$, ou seja, houve ganho de $\mathrm{R}$ \$5,00/@. Observa-se que a utilização de animais da raça Nelore no confinamento proporcionou maiores prejuízos para o sistema de produção, uma vez que estes animais apresentaram maior consumo diário de matéria seca $(10,17$ vs. $9.88 \mathrm{~kg} /$ dia) e menor ganho de peso médio diário.

A avaliação com a aquisição de animais demonstra que o confinamento também é atividade especulativa, ou seja, o retorno financeiro está em função dos valores de compra e venda dos animais. Todavia, como já mencionado, é possível o produtor evitar a especulação por meio de contratos futuros de compra e venda.

Segundo Oliveira Neto e Figueiredo (2008), a dificuldade dos produtores de bovinos de corte em lidar com cenários incertos de formação de preços no mercado físico, assim como a necessidade real em se proteger contra as constantes oscilações de preços, demonstra a importância das operações de hedge em mercados futuros. No trabalho realizado por estes autores, concluiu-se que as operações de hedge da arroba do boi gordo para o estado de Goiás representou uma diminuição de aproximadamente $90 \%$ do risco. Espera-se que comportamento semelhante seja encontrado para a região sul de Minas Gerais.

É importante destacar que não se pode avaliar a atividade de confinamento de forma isolada. No contexto global da empresa rural, este proporciona aumento na taxa de lotação da propriedade e, como consequência, o aumento do ganho de peso vivo por hectare, que pode elevar a rentabilidade da atividade e o capital de giro. Além disso, de acordo com Lanna e Almeida (2005) a retirada dos animais mais pesados da pastagem permite melhorar a disponibilidade de forragem para as outras categorias do rebanho e/ou reduzir a lotação dos pastos no início da primavera, o que aumenta a produtividade dessas no inicio do verão, além de diminuir despesas com reformas de pastagens degradadas. 
Tabela 3 - Avaliação econômica do confinamento de novilhos Nelore e Red Norte, considerando a aquisição de animais.

\begin{tabular}{lcccc}
\hline \multirow{2}{*}{ Especificação } & \multicolumn{2}{c}{ Red Norte } & \multicolumn{2}{c}{ Nelore } \\
\cline { 2 - 5 } & \multicolumn{2}{c}{ Total } & \multicolumn{2}{c}{ Total } \\
& $\mathrm{R} \$ /$ animal & $\mathrm{R} \$ / @$ & $\mathrm{R} \$ /$ animal & $\mathrm{R} \$ / @$ \\
\hline Renda bruta & & & & \\
1.1 Arrobas produzidas comercializadas & 394,80 & 60,00 & 338,40 & 60,00 \\
1.2 Arrobas existentes na aquisição comercializadas & 643,80 & 60,00 & 631,80 & 60,00 \\
Renda Bruta Total & 1038,60 & 60,00 & 970,20 & 60,00 \\
Custo de produção & & & & \\
Custo Total & 444,86 & 25,42 & 454,47 & 27,81 \\
Custo com a aquisição de animais & 586,0 & 34,08 & 578,0 & 35,80 \\
CA da aquisição de animais & 10,86 & 0,63 & 10,66 & 0,66 \\
Custo Total c/ aquisição & 1040,95 & 60,14 & 1039,31 & 64,27 \\
\hline Resultado & $-2,35$ & $-0,14$ & $-69,11$ & $-4,27$ \\
\hline
\end{tabular}

Silveira et al. (2010) realizaram uma simulação para verificar a área de pastejo necessária para o abate anual de 1680 bovinos em três sistemas de produção: abate aos 13, 24 ou 33 meses. Verificou-se que, para o mesmo número de matrizes, o sistema com abate aos 13 meses, em virtude da supressão da recria, exige bem menos área de pasto em relação aos outros sistemas, para se abater igual número de bezerros. Segundo os autores, para produzir o mesmo número de bezerros com abate aos 24 meses, há necessidade de aumento da área disponível em 79\%, já para o sistema que visa a idade de abate aos 33 meses, há necessidade de aumento de $121 \%$. Diante do exposto, com a redução da idade de abate, que pode ser obtida com o uso do confinamento, o produtor pode aumentar significativamente o número de matrizes da propriedade e aumentar a produtividade por área.

De acordo com os dados obtidos, fica evidente que o confinamento, como atividade exclusiva, apenas será benéfico em situações particulares, como por exemplo: para frigoríficos que se encontram em regiões onde a seca prolongada acarreta grandes dificuldades para a obtenção de animais com grau de acabamento adequado; ou quando os preços de venda da arroba estiverem elevados, se comparados aos custos com alimentação e aquisição de animais. Apesar de não ser tão marcante quanto no passado, a variação de preço do valor da arroba na safra e entressafra, em alguns anos, pode permitir que o confinamento como atividade exclusiva seja lucrativo.

\section{CONCLUSÃO}

A viabilidade econômica da terminação em confinamento na região de Lavras, sul do estado de Minas Gerais, pode apresentar resultados variáveis em função dos custos com a alimentação e preços de aquisição e venda dos animais. Todavia, a utilização de animais mais eficientes nutricionalmente pode permitir a redução dos custos e melhorar a lucratividade.

\section{REFERÊNCIAS BIBLIOGRÁFICAS}

AMARAL, J.J.F. Sustentabilidade: preparando a gestão sustentável. Novo Hamburgo: Fundação Semear, 2010. Disponivel em: <http://www2. fundacaosemear.org.br Acesso em: 6 dez. 2010.

\section{BARBOSA, F.A.; SOUZA, R.C. Administração de}

fazendas de bovinos: leite e corte. Viçosa, MG: Aprenda Fácil, 2007. 342p.

BONILHA, S.F.M.et al. Efeitos da seleção para peso pósdesmama sobre características de carcaça e rendimento de cortes cárneos comerciais de bovinos. Revista Brasileira de Zootecnia, Viçosa, v.36, n.5, p.1275-1281, 2007.

CALEGARE, L. et al. Preweaning performance and body composition of calves from straightbred Nellore and Bos taurus x Nellore crosses. Journal of Animal Science, Chanpaign, v.87, p.1814-1820, 2009. 
CAMARGO, S.H.C.R.V. et al. varejo de carnes: um estudo multicaso no interior de São Paulo. Interface Tecnológica, São Paulo, v.5, p.57-75, 2008.

FERREIRA, I.C.; SILVA, M.A.; REIS, R.P. Análise de custos de diferentes grupos genéticos de bovinos de corte terminados em confinamento. Arquivo Brasileiro de Medicina Veterinária e Zootecnia, Belo Horizonte, v.56, n.3, p.385-391, 2004.

INSTITUTO BRASILEIRO DE GEOGRAFIA E ESTATÍSTICA. Indicadores de produção agropecuária 2007. Disponivel em: বhttp:///www. ibge.gov.br/homè. Acesso em: 27 abr. 2008.

LANNA, D.P.D.; ALMEIDA, R. A terminação de bovinos em confinamento. Visão Agrícola, Piracicaba, n.3, p.55$58,2005$.

LOPES, M.A.; MAGALHÃES, G.P. Análise da rentabilidade na terminação de bovinos de corte em confinamento: um estudo de caso. Arquivo Brasileiro de Medicina Veterinária e Zootecnia, Belo Horizonte, v.57, n.3, p.374-379, 2005.

NATIONAL RESEARCH COUNCIL. Nutrients requeriments of dairy cattle. 7.ed. Washington, 2001. 244p.

NOGUEIRA, M.P. Gases da pecuária: o outro lado da moeda. São Paulo: Boi \& Companhia, 2006. 14p.

OLIVEIRA, R.L.; ASSUNÇÃO, D.M.P.; BARBOSA, M.A.A.F. Desempenho produtivo e custos com alimentação de novilhos bubalinos alimentados com dietas com diferentes fontes de lipídeos. Revista Brasileira de Zootecnia, Viçosa, v.36, n.3, p.727-732, 2007.

OLIVEIRA NETO, O.J.; FIGUEIREDO, R.S. Análise das operações de hedge do boi gordo no mercado futuro da BM\&F para o estado de Goiás. Revista Gestão e Planejamento, Salvador, v.9, n.1, p.77-93, 2008.

PACHECO, P.S.; RESTLE, J.; VAZ, F.N. Avaliação econômica da terminação em confinamento de novilhos jovens e superjovens de diferentes grupos genéticos. Revista Brasileira de Zootecnia, Viçosa, v.35, n.1, p.309-320, 2006.

REIS, R.P. Abate de animais, produção de leite, couro e ovosAbate de animais, produção de leite, couro e ovos Fundamentos de economia aplicada. Lavras: UFLA/ FAEPE, 2002. 95p.

RESTLE, J.; VAZ, F.N. Confinamento de bovinos definidos e cruzados. In: LOBATO, J.F.P. et al. (Eds.). Produção de bovinos de corte. Porto Alegre: EDIPUCRS, 1999. p.141-168.

RODRIGUEZ, N.M.; SALIBA, E.O.S.; GUIMARÃES, J.R.R. Uso de indicadores para estimar consumo y digestibilidad de pasto: lipe, lignina purificada y enriquecida. Revista Colombiana de Ciências Pecuárias, Bogotá, v.20, p.518-525, 2007.

SILVEIRA, A.C. et al. Produção de bovino superprecoce no Brasil. In: PIRES, A.V. (Org.). Bovinocultura de corte. Piracicaba: ESALQ, 2010. p.1347-1368. 